\title{
O call na aprendizagem da habilidade de escrita de língua inglesa
}

\author{
Sheila Cristina Alves de Ávila \\ Universidad Americana - Paraguai \\ Anderson Luiz da Silva \\ EPCAR - MG
}

\begin{abstract}
Resumo
O uso das tecnologias e sua adoção como aliadas no processo ensinoaprendizagem é cada vez mais frequente, principalmente daquelas que circulam via internet, na busca de tornar o ensino mais dinâmico, eficaz e acessível para um maior número de pessoas. As teorias relativas à aquisição de uma língua estrangeira podem auxiliar na elaboração de um projeto de execução de CALL (Computer Assisted Language Learning - Aprendizado de Língua Mediado por Computador) no aprimoramento das habilidades linguísticas requeridas para atingir proficiência: leitura, escrita, compreensão auditiva e fala. Tecnologias podem ser utilizadas como ferramentas de apoio na aprendizagem da habilidade de escrita de Inglês como língua estrangeira no processo de aquisição da língua (SLA - Second Language Acquisition), estimulada pela leitura ${ }^{1}$. Este artigo relata uma pesquisa que visa alcançar ganhos nessa prática, delimitando-a e estabelecendo comparação com o não-uso do CALL.
\end{abstract}

Palavras-chave: CALL, SLA, teorias de aquisição de língua estrangeira, escrita.

\begin{abstract}
Technology has been adopted more and more frequently in the process of learning and teaching, principally the technological resources which are found on Internet and are used in the attempt to make teaching more dynamic, efficient and accessible to a larger number of people. Theories of foreign language acquisition may provide the basis for a CALL (Computer Assisted Language Learning) project that seeks to improve the linguistic abilities required for achieving proficiency in reading, writing, listening and speaking. Technology can also be used as a support tool for learning writing skills in English, stimulated by reading, during the process of language acquisition (SLA - Second Language Acquisition). This article reports on some research which aims to improve this practice, delimiting it and drawing comparisons with teaching where CALL is not adopted. Key-words: CALL, SLA, foreign language learning theories, writing.
\end{abstract}

1 Entenda-se leitura no sentido amplo, incluindo material verbal ou não, utilizado como suporte para estimular a produção escrita: textos, vídeos, imagens, etc. 


\section{INTRODUÇÃO}

Este artigo apresenta resultados preliminares de uma pesquisa acerca da habilidade de escrita do Inglês como língua estrangeira, iniciada em fevereiro de 2011 e com término previsto para julho do mesmo ano. Pretende-se fazer análise e correlação dos aspectos da habilidade através da abordagem da aprendizagem humanística generalizada (Teoria Associativo-cognitiva, Teoria de Aquisição de Habilidade), buscando, assim, resultados diagnósticos, por meio de uma pesquisa de campo. Trata-se de um estudo descritivo do desempenho de dois grupos de alunos (grupo de pesquisa e de controle) do nível pósintermediário de língua inglesa do CPCAR (Curso Preparatório de Cadetes do Ar), curso de ensino médio oferecido pela EPCAR (Escola Preparatória de Cadetes do Ar), pertencente ao Comando da Aeronáutica, em Barbacena, estado de Minas Gerais, Brasil.

Por meio das ferramentas e-mail e blog, visa-se ao aprimoramento da habilidade de escrita dos pesquisados e à verificação se o mesmo ocorre com o grupo de controle, caracterizando o trabalho, quanto ao uso do tempo, como um estudo experimental clássico, no qual um grupo experimental é especialmente criado e submetido a um estímulo com o intuito de ser comparado a um grupo de controle (privado do estímulo). Logo, há uma abordagem de alcance qualitativo, cujas unidades de observação são tratadas de maneira intensiva, com predomínio da expressão livre do participante e com número reduzido de casos observados (amostragem). Os dados obtidos deverão ser analisados indutivamente e a eles atribuídos significados e interpretações. A habilidade será praticada com o auxílio da tecnologia, com base nas categorias de texto requeridas pelo exame FCE (First Certificate in English), nível B2 de acordo com os requisitos estabelecidos pelo CEFR $^{2}$. Será mensurada a evolução da escrita dos alunos através dos padrões requeridos, comparando os resultados entre os dois grupos acima citados. Serão estabelecidas as devidas conexões entre as teorias de aquisição de língua estrangeira e CALL e também descritos os mecanismos usados como fundamento para a avaliação do processo, tais como padrões de referência e exames.

Acredita-se que o CALL possa auxiliar no aprimoramento dessas habilidades através da prática e da interação via ferramentas que a tecnologia proporciona. Assim, optou-se pela utilização das ferramentas blog e e-mail como auxílio e ambiente de interação entre professor e aluno, aluno e aluno e/ou aluno e computador; como fonte de estímulo e meio de divulgação de algumas informações necessárias à evolução da habilidade trabalhada; instrumento de

\footnotetext{
2 CEFR - Common European Framework of Reference for Languages (Sistema Comum Europeu de Referência
} para Línguas), o qual aparece em diferentes fontes com duas abreviaturas: CEFR ou CEF. 
veiculação da retroinformação (feedback) do professor, auxílio que se acredita importante para a análise e crescimento linguístico do aluno na aquisição da habilidade requerida.

Objetiva-se, assim, analisar o aprendizado da habilidade de escrita em inglês como língua estrangeira por meio do CALL e observar se há ganhos nesta prática, para assim delimitá-los e estabelecer comparação com o não-uso do CALL. Para isso, serão considerados: a) a prática da habilidade de escrita com o auxílio da tecnologia, usando $e$-mail e blog como ferramentas, com base nas categorias de texto requeridas pelo exame FCE, oferecido por Cambridge $\mathrm{ESOL}^{3}$ Examinations, uma subunidade da Universidade de Cambridge, nível B2, de acordo com os requisitos estabelecidos pelo CEFR; b) a mensuração da evolução da escrita dos alunos através dos padrões requeridos; c) a comparação dos resultados entre grupo de pesquisa e grupo de controle. ${ }^{4}$

Considerando a trajetória do CALL e a estreita relação que pode ser estabelecida entre seus pressupostos e as teorias de aquisição de língua estrangeira (SLA), este artigo visa, sobretudo, relacionar teorias de aquisição de língua estrangeira com o aprendizado mediado por computador, pois abordagens teóricas se fazem necessárias no desenvolvimento e avaliação de materiais e tarefas, e fundamentam, norteiam os caminhos pelos quais se pleiteia buscar a aprendizagem.

\section{CALL - COMPUTER ASSISTED LANGUAGE LEARNING}

O CALL surgiu com o advento e propagação deste equipamento, principalmente na década de 1980, e vem acompanhando as possibilidades que o computador e as ferramentas que dele provêm podem oferecer para buscar inovações, estímulos e/ou práticas que se julguem necessárias para melhorar os resultados do processo ensino-aprendizagem. No final dos anos 1960 e nos anos 1970, havia o aprendizado chamado Computer Assisted Instruction (Instrução Assistida pelo Computador), computadores com tecnologia bastante rudimentar e memória com capacidade reduzida. Além destas denominações, existem diferentes acrônimos para se referir ao aprendizado com o auxílio da tecnologia, por exemplo, CMC (Computer Mediated Communication - Comunicação Mediada por Computador), TMLL (Technology Mediated Language Learning - Aprendizado de Língua Mediado pela Tecnologia), TELL

\footnotetext{
${ }^{3}$ ESOL - English for Speakers of Other Languages (Inglês para falantes de Outras Línguas).

${ }^{4}$ A comparação entre os grupos está incluída no propósito da pesquisa, porém, neste artigo, por se tratar de análise ainda preliminar de resultados, não será levada em conta.
} 
(Technology Enhanced Language Learning - Aprendizado de Língua Intensificado pela Tecnologia) (Otto \& Pusack, 2009). Crystal (2010) cita que, no seu aspecto de comunicação eletrônica, o nome CALL tem suas limitações, por isso outros nomes foram sugeridos para substituí-lo, mas que esta terminologia permanece liderando a nomenclatura deste tipo de estudo. Autores como Brown (2007), Chapelle (2009), Crystal (2010), Lafford (2009), Otto \& Pusack (2009) defendem a universalidade do termo consagrado e o consideram abrangente o bastante para denominar o uso da tecnologia associada à aquisição ou aprimoramento de habilidades em língua estrangeira.

Inicialmente, o CALL se baseava apenas no contato entre o aprendiz e o programa de computador que continha instruções e prática das habilidades linguísticas (Garret, 2009a). ${ }^{5}$ Atualmente, o conceito de CALL aparece mais abrangente, tanto no que se refere à interação dos estudantes com os programas, quanto à possibilidade de que o professor se utilize das ferramentas apropriadas ao seu objetivo de ensino. A internet, por sua vez, propicia uma variedade de conteúdo falado, escrito e gráfico sem precedentes (salas de bate-papo, sites de redes sociais, ambientes de aprendizado virtual, e-mail, mensagens instantâneas, a participação em tweets, blogs, etc.), propiciando novas e motivadoras oportunidades de interação entre alunos, destes com seus professores ou com nativos da língua estudada. Consequentemente, a evolução da rede (web) facilita a comunicação online, a colaboração, a troca de informações e as simulações virtuais (Crystal, 2010; Otto \& Pusack, 2009).

Sobre o uso das ferramentas e-mail e blog, ambas utilizadas na pesquisa que este artigo relata, Brown (2007, p.203-204) trata da primeira como um ambiente que "[...] propricia oportunidades para leitura e escrita sobre tópicos de interesse." ${ }^{6}$ Sobre a segunda, afirma que "o uso dos blogs (blogging) tem se tornado muito popular, especialmente entre os jovens. Blogs são sites simples e fáceis de usar, onde se pode escrever pensamentos rapidamente, interagir com outros, obter retroinformação (feedback), postar fotos, entre outros". (tradução nossa $\left.{ }^{7}\right)^{8}$

Em 1991, numa realidade bem diferente da atual, com recursos tecnológicos e interação via computador limitados, Garret já ressaltava a importância da concepção de que

\footnotetext{
${ }^{5}$ Artigo publicado pela primeira vez no periódico The Modern Language Journal (1991), 75, 74-101. Republicação do artigo na íntegra em 2009. Vide referências.

${ }^{6}[\ldots]$ provide opportunities for reading and writing on topics of interest.

${ }^{7}$ No original: "The use of blogs (blogging) has become very popular, especially among young people. Blogs are easy-to-use simple web sites where one can quickly write thoughts, interact with others, get feedback, post photos, and more." Esta, como as demais traduções que aparecem ao longo desde artigo, são de autoria da pesquisadora Sheila Ávila, e possuem apenas finalidade didática para o presente trabalho.

${ }^{8} \mathrm{O}$ blog <http://www.projetocallsheila.blogspot.com> foi criado para disponibilizar atividades da pesquisa via internet.
} 
“[...] o uso do computador não constitui um método, mas um meio ou um ambiente no qual uma variedade de métodos, abordagens ou filosofias pedagógicas podem ser implementados." "9 (2009a, p. 698). A autora vê a necessidade do estabelecimento de algumas prioridades para a próxima década, entre elas a preocupação com a individualidade do aprendiz, a integração entre o ensino de língua, literatura e cultura e também entre teoria e prática. E, ainda, a necessidade de se equilibrar os três componentes principais do CALL pedagogia, teoria e tecnologia (Garret, 2009b) - sugerindo a inclusão de um quarto componente, a infra-estrutura, ou seja, o ambiente que afeta a maneira como os outros três componentes se integram.

\section{TEORIAS DE AQUISIÇAO DE LÍNGUA ESTRANGEIRA (SLA)}

Chapelle (2009) estabelece conexões entre as teorias de SLA e CALL, agrupando as perspectivas teóricas em quatro abordagens: linguística cognitiva (Gramática Universal, Teoria da Indução Autônoma, Abordagem Orientada pelo Conceito); psicolinguística (Teoria da Processabilidade, Abordagem de Processamento por Estímulo, Abordagem Interacionista); aprendizagem humanística generalizada (Teoria Associativo-cognitiva, Teoria de Aquisição de Habilidade); e língua no contexto social (Abordagens: Sócio-cultural, Socialização da Língua, Análise do Discurso, Funcional-sistêmica e Teoria da Complexidade). ${ }^{10}$

Como o processo de aprendizagem linguística não é linear, esta característica dificulta o estabelecimento de critérios rígidos para delimitá-lo ou avaliá-lo. Além disso, vários aspectos influem no seu resultado, uma vez que se trata de indivíduos inseridos em um contexto social e com experiências de aprendizagem diversas. Entretanto, este artigo pretende registrar, analisar e correlacionar aspectos da habilidade de escrita através da aprendizagem humanística generalizada (Associative-cognitive CREED, Skill Acquisition Theory), verificando, através da análise dos textos produzidos, se há aprimoramento no desempenho dos alunos.

\footnotetext{
9 [...] the use of the computer does not constitute a method. The computer is rather a medium or an environment in which a wide variety of methods, approaches or pedagogical philosophies may be implemented.

${ }^{10}$ Termos traduzidos da Língua Inglesa. Nomenclatura original: cognitive linguistic (Universal Grammar, autonomous induction theory, the concept-oriented approach); psycholinguistic (processibility theory, input processing theory, interactionist theory); human learning (associative-cognitive CREED, skill acquisition theory); and language in social context (sociocultural, language socialization, conversation analysis, systemicfunctional, complexity theory) (Chapelle, 2009, p. 741)
} 


\section{ASSOCIATIVE-COGNITIVE CREED}

A abordagem associativo-cognitiva CREED assume que a aquisição da língua estrangeira é regida pelos mesmos princípios de aprendizagem associativa e cognitiva que sustentam qualquer conhecimento humano. A aprendizagem associativa foi inicialmente adotada pela tradição behaviorista, fundamentada em estímulo e resposta, ocupando-se unicamente das instâncias do comportamento, deixando a cargo da psicologia qualquer questão ligada à percepção, à análise (Skinner, 1989). Os processos de aprendizagem estudados pela psicologia cognitiva incluem aspectos mais conscientes, explícitos, dedutivos ou monitorados (N. Ellis, 2006, 2008), para os quais o conhecimento do assunto é mais determinante para o sucesso da tarefa, que consiste em resolver problemas usando o intelecto em vez da destreza física (Vanlehn, 1996).

Segundo a abordagem associativo-cognitiva (N. Ellis, 2006, 2008), os princípios que regem a aquisição da língua estrangeira são construtivos, racionais, guiados pelo exemplo, emergentes e dialéticos. Os princípios acima citados foram sintetizados no acrônimo CREED (Construction-based, Rational, Exemplar-driven, Emergent, and Dialetic). A competência é conseguida por uma contextualização dinâmica, na qual a primeira língua (L1) tem influência direta, racionalizando o aprendizado e criando tensão dialética entre a evolução da interlíngua (interlanguage) e as barreiras criadas pela língua materna. N. Ellis $(2006,2008)$ sugere que a retroinformação (feedback), seja ela de natureza linguística, pragmática ou metalinguística, permite o desenvolvimento scaffolded, isto é, o suporte dado aos aprendizes lhes propicia a execução de tarefas que estão acima da sua capacidade. Inicialmente, no aprendizado linguístico, eles podem ser incapazes de produzir certas estruturas ou expressões, mas podem construí-las através da interação. (Richards \& Schmidt, 2002, p.466)

O termo interlíngua, citado anteriormente, foi criado por Selinker e representa o sistema linguístico alternativo criado pelo aprendiz, uma gramática mental, diferente daquele sistema existente na sua primeira língua (L1) ou na língua alvo (L2), fundamentada nas experiências que permeiam as duas línguas envolvidas no processo de aprendizagem e na influência externa, através de estímulos recebidos. A interlíngua é baseada nas experiências do aprendiz com a L2 e consiste em transferências da língua materna, estratégias de aprendizagem, estratégias de comunicação e generalização dos padrões da língua alvo (R. Ellis, 2003). O pesquisador (2003, p.33) descreve a interlíngua como:

[...] um processo transitório em que os aprendizes mudam sua gramática de tempo em tempo, adicionando regras, excluindo regras, reestruturando todo o sistema. Isso resulta em uma interlíngua contínua, ou seja, aprendizes constroem séries de gramáticas mentais ou 
interlínguas na medida em que gradualmente aumenta a complexidade do seu conhecimento da L2. ${ }^{11}$

O Princípio Construtivo (Construction-based) pressupõe que as unidades de representação da língua são construções, mapas de significação de sistemas, de natureza simbólica, que foram convencionalizados numa referida comunidade linguística e que se formalizaram como conhecimento linguístico na mente do aprendiz (N. Ellis, 2006, 2008). As construções são os componentes principais da linguística cognitiva e das teorias funcionais da linguagem, aprendidas através do uso da língua e da comunicação. $\mathrm{O}$ autor afirma que a competência linguística individual emerge da conexão entre a nossa memória de tudo o que usamos de determinada língua e da abstração da frequência de regularidades entre aspectos desse uso. Segundo ele, as construções variam de simples itens lexicais a complexas fórmulas, que aprendemos pela repetição e pela probabilidade, devido ao fato de que a cognição humana é fundamentalmente probabilística. Assim, quanto maior for a frequência em que determinadas construções ocorrem (letras, morfemas, padrões sintáticos, etc.), mais rápido será o seu aprendizado pela associação e pelo uso. Os mapas de significação partem da forma para a interpretação, estabelecendo uma rede de associações somadas pela experiência, ou seja, uma rede semântica que compreende o conhecimento linguístico do falante. Para N. Ellis (2006, 2008), outros fatores contribuem para essa significação individual; são eles: pistas, principalmente, acrescidas de contexto, que lhes confere maior sentido por combinarem probabilidade a sequência, facilitando a interpretação.

O Princípio Racional do processamento linguístico (Rational) caracteriza o aprendizado de uma língua como uma constante solução de problemas, com aspectos intuitivos e estatísticos, isto é, o aprendiz deve solucionar o que a língua lhe apresenta e otimizar a comunicação através de uma análise racional, considerando: a frequência, o que é recente e o contexto das construções (N. Ellis, 2006, 2008). Os três componentes são considerados pelo autor como de fundamental influência na cognição humana, seja ela linguística ou não-linguística.

Quanto ao Princípio Guiado pelo Exemplo (Exemplar-driven), N. Ellis $\quad$ (2006, 2008) ressalta que, embora o uso da língua seja basicamente repetitivo (formulaic), baseado em fórmulas predefinidas a serem memorizadas pelo aprendiz, existe uma parte que necessita da interpretação, processamento do falante, objeto de estudo das teorias fundamentadas no

\footnotetext{
11 The learner's grammar is transitional. Learners change their grammar from time to time to another by adding rules, deleting rules, and restructuring the whole system. This results in an interlanguage continuum. That is, learners construct a series of mental grammars or interlanguages as they gradually increase the complexity of their L2 knowledge.
} 
uso da língua (usage-based theories). Tais teorias investigam como a aquisição desses padrões produtivos (generative schema) ${ }^{12}$ e outras regularidades linguísticas são fundamentas em exemplos através da inferência indutiva do aprendiz. Isto significa que quando nos deparamos com exemplares mais complexos do que aqueles aos quais estamos habituados, ativamos os exemplares que temos armazenados, generalizamos, fundamentados na frequência. Deste modo, da língua emergem "protótipos, exemplares que são mais típicos de suas categorias, aqueles que são similares a muitos membros da sua categoria, mas não são similares aos membros de outras categorias"13 (N. Ellis, 2008, p.80). O autor também afirma que, de acordo com a teoria cognitiva, os mesmos padrões humanos utilizados para o reconhecimento, categorização e cognição racional são igualmente aplicados em relação à língua, que é classificada, conforme o fazemos em outras tantas situações cotidianas.

Para tratar do Princípio emergente (Emergent), é necessário primeiro definir duas abordagens: o emergentismo e o conexionismo. O primeiro apresenta "[...] a visão de que formas de cognição mais completas emergem da interação entre formas mais simples de cognição e a arquitetura do cérebro humano" ${ }^{14}$ (Richards \& Schmidt, p. 177, 2002). Quanto ao conexionismo, ele

[...] assume que os componentes individuais da cognição humana são altamente interativos e que o conhecimento de eventos, conceitos e língua são difundidos no sistema cognitivo [...] criando modelos matemáticos e simulações computacionais que tentam capturar tanto a essência do processamento de informação quanto dos processos de pensamento ${ }^{15}$ (Richards \& Schmidt, p. 108, 2002).

O aprendizado associativo permite, através dos mecanismos de racionalidade, análise do que é estímulo recebido (input) e, pelo uso, que todo indivíduo adquira fluência na sua língua materna (L1). Entretanto, o aprendizado de uma segunda língua (SLA) não logra igual sucesso. Existe, neste contexto, distinção entre o estímulo recebido, segundo Corder (1967, apud N. Ellis, 2008), classificado como qualquer material disponível da língua alvo (input) e somente aquilo que faz sentido e será utilizado pelo aprendiz (intake). Ciente das limitações

12 Schema theory - teoria de acordo com a qual na medida em que o falante compreende a língua, ativa schemata relevante, ou seja, significa a informação recebida de acordo com a sua experiência linguística com o intuito de processar e interpretar novas experiências de maneira rápida e eficiente. (Richards \& Schmidt, p. 469, 2002)

13 Prototypes, exemplars that are the most typical of their categories, are those that are similar to many members of their category but are not similar to members of other categories.

$14[\ldots]$ the view that higher forms of cognition emerge from the interaction between simpler forms of cognition and the architecture of the human brain.

15 [...] assumes that the individual components of human cognition are highly interactive and that knowledge of events, concepts and language is represented diffusely in the cognitive system [...] provides mathematical models and computer simulations that try to capture both the essence of information processing and thought processes. 
presentes no aprendizado de uma língua estrangeira, ou segunda língua ${ }^{16}$, a teoria do aprendizado associativo busca explicar tais limitações à luz dos aspectos associativos da transferência, que são a atenção aprendida e a interferência positiva da L1. Para esta teoria, as experiências com a língua materna facilitam o aprendizado da L2 pela indução associativa. (N. Ellis, 2006, 2008)

Segundo o Princípio dialético (Dialetic), no aprendizado associativo de uma língua estrangeira (L2) geralmente o falante não atinge um estágio próximo daquele em que se encontra o falante nativo, mas permanece em um estágio básico de interlíngua, suficiente apenas para uma comunicação diária, sem a presença de elementos mais elaborados da língua em seu discurso (Klein, 1998 apud N. Ellis, 2006). O processo de aquisição de uma língua estrangeira é dialético porque o aprendiz se vê envolvido em uma tensão entre a sua interlíngua e o feedback recebido, que pode levá-lo ao desenvolvimento social scaffolded (além do que iria sozinho), na busca de atingir uma comunicação mais elaborada e fluente, próxima daquela que o falante nativo possui.

\section{SKILL ACQUISITION THEORY}

Os fundamentos científicos da teoria de aquisição de habilidade se encontram na psicologia, passando pelo behaviorismo, o cognitivismo e o conexionismo. Segundo Vanlhen, a aquisição de habilidade cognitiva fundamenta-se historicamente nos estudos de solução de problemas, iniciados por Duncan (1959), continuados através da análise da solução de enigmas e quebra-cabeças na década seguinte e com os estudos de Newell e Simon (1972), os quais introduziram muitos conceitos teóricos importantes; nos anos 1980, os estudos se concentraram na automatização e, sucessivamente, buscou-se conhecer os estágios iniciais da aquisição da habilidade e o papel dos exemplos. Seus pesquisadores se concentram em como tarefas complexas, de conhecimento intensivo, são desempenhadas satisfatoriamente e como se adquire automatização em determinada habilidade (Vanlhen, 1996).

A teoria concebe que existe um conjunto de princípios básicos comuns à aquisição de qualquer habilidade (skill), que vão do desenvolvimento a partir da representação inicial do conhecimento, das mudanças que se seguem no comportamento do aprendiz, à espontaneidade daquele que já está altamente qualificado em determinada habilidade (Dekeyser, 2008). De acordo com Dekeyser, estes três estágios de desenvolvimento recebem

\footnotetext{
${ }^{16}$ A sigla SLA se refere tanto à segunda língua quanto à língua estrangeira, entendidas e usadas aqui como termos equivalentes, indicando uma língua além da língua materna do aprendiz.
} 
diferentes nomenclaturas por autores diversos. São respectivamente: cognitivo, associativo e autônomo (Fitts \& Posner, 1967); declarativo, processual e automatizado (Anderson, 1982,1993; Anderson et al, 2004); apresentação, prática e produção (Byrne, 1986). ${ }^{17}$

Os três estágios da aquisição de uma habilidade apresentam características e períodos de duração distintos. Porém, é importante lembrar que a divisão em três fases cronológicas descrita nesta pesquisa é uma idealização, cujas fronteiras não são lineares, e que em dado momento os alunos podem possuir componentes de mais de uma fase (Vanlehn, 1996).

No primeiro estágio (declarativo), o estudante aprende principalmente pela observação e análise de outras pessoas que possuem aquela habilidade. O aprendiz não tem desenvoltura na sua execução ou apenas busca conhecer a sua teoria. No segundo estágio (processual) o conhecimento é transformado em comportamento, mudando de "que" para "como", ou de conhecimento factual para processual. Trata-se de um estágio relativamente curto uma vez que o estudante já possui o conhecimento, apenas necessita aplicá-lo. Completo o estágio intermediário, segue um longo caminho até que o estudante consiga fluência, espontaneidade e reduza ao mínimo os erros cometidos. Assim, para Dekeyser, nesta fase do aprendizado

[...] o conhecimento ainda não é robusto e refinado. Uma grande quantidade de prática é necessária para diminuir o tempo requerido para a execução da tarefa (tempo de reação), a porcentagem de erros (índice de erros) e a quantidade de atenção requerida (e, portanto, interferência com/de outras tarefas). Esta prática leva à gradual automatização do conhecimento $^{18}$ (2008, p. 98,99).

O estágio processual é marcado pela presença de exemplos, pois se caracteriza pela fase na qual a solução de problemas se efetiva, e "antes que eles comecem a solucionar problemas sozinhos, eles geralmente estudam alguns problemas que já foram solucionados (denominados exemplos de agora em diante)" ${ }^{\prime 19}$ (Vanlehn, 1996, p. 516). O autor afirma que pesquisas demonstram que muitos alunos preferem aprender através de exemplos a outras possíveis maneiras de lidar com o aprendizado e que, devido à sua importância, exemplos são abundantes em muitos materiais destinados a esta fase intermediária do aprendizado. Logo, cabe dizer que nos livros didáticos, geralmente os assuntos são treinados, praticados a partir de exemplos que os constituem ou deles fazem parte para a posterior solução de problemas. A

${ }^{17}$ Será adotada, neste estudo, a nomenclatura: declarativo, processual e automatizado.

18 [...] the knowledge is not yet robust and fine-tuned. A large amount of practice is needed to decrease the time required to executate the task (reaction time), the percentage of errors (error rate), and the amount of attention required (and hence interference with/from other tasks. This practice leads to gradual automatization of knowledge.

19 Before they begin solving problems themselves, they often study a few problems that have already been solved (called examples henceforth). 
resolução é facilitada pelo modelo ou exemplo previamente conhecido, com o intuito de facilitar a correspondência entre eles, problema e exemplo, pela inferência. O exemplo suscita outros processos, tais como, recuperação de informação (retrieval), mapeamento (mapping), aplicação (application) e generalização (generalization) (Vanlehn, 1996).

A recuperação de informação pode ser espontânea ou deliberada, no segundo caso, quando há pistas explícitas ou simulação que ligarão diretamente o exemplo à resolução do problema; no primeiro, também conhecido como reminding (algo que traz à memória), a ligação entre exemplo e problema é implícita. A recuperação de informação deliberada é considerada mais eficaz pela sua característica de conexão direta, de similaridade. O mapeamento é o processo através do qual as partes do exemplo se conectam às partes do problema. A aplicação consiste em verificar se o mapeamento está correto através da utilização ou não do exemplo na sua resolução. A generalização permite que se aplique o exemplo a outros problemas, que se expanda a habilidade aprendida (Vanlehn, 1996).

Neste estágio marcado por exemplos, alunos que conseguem um bom aprendizado são aqueles que entendem os exemplos e princípios, aprendendo com os próprios erros e analisando a sua produção, enquanto os que têm desempenho insatisfatório apenas confiam nos exemplos, utilizando-os sem entendê-los de fato (Chi et al, 1989). A respeito da retroinformação, ela pode ser mínima (minimal feedback) ou mais direta (feedback). A primeira não especifica exatamente os erros, apontando-os ou corrigindo-os, mas trata-os de maneira genérica; a outra acredita que agir de maneira mais explícita em relação aos erros é o melhor caminho para evitar que se repitam. Há divergência entre autores sobre qual seria o melhor caminho para minimizar os erros dos aprendizes (Vanlehn, 1996). Vanlenh conclui que estudos mostram que na maioria das vezes os erros são causados pela falta de conhecimento mais do que pelo conhecimento incorreto, porque, ao invés de buscar ajuda, seja ela de qualquer natureza, o aprendiz opta por inventar o que lhe convier no momento da execução da tarefa a fim de dar-lhe continuidade (1990, 1995 apud Vanlehn, 1996).

O estágio autônomo ou automatizado é marcado pelos efeitos da prática. A nova fase se caracteriza pela produção com o mínimo de erros, cuja prática visa aumentar velocidade e precisão. $\mathrm{O}$ conceito central do estudo da aquisição da habilidade se pauta no que denominam como lei poderosa do aprendizado ou da prática (power law of learning/practice), isto é, tanto o tempo de reação quanto o índice de erros são reduzidos através da prática. E a aparente mudança quantitativa, é, na verdade uma mudança de ordem qualitativa dos mecanismos de ativação do conhecimento, que não atingirá seu ápice absoluto (Logan, 1988; Vanlehn, 1996; Dekeyser, 2008). Em termos de aquisição de língua 
estrangeira, pode-se dizer que o desempenho semelhante àquele do falante nativo não será atingido, mas se conseguirá chegar bem próximo desse padrão tido como ideal. Para Logan (1988), o processamento é considerado automático na medida em que consegue recuperar o conhecimento armazenado através da prática, que terá como consequências o aumento da velocidade dessa ativação do conhecimento previamente adquirido bem como a qualidade da produção (redução do desvio do padrão de língua esperado).

\section{METODOLOGIA}

O objeto de estudo é constituído por textos produzidos pelos alunos pertencentes aos grupos de pesquisa e de controle. A pesquisa se pauta no material escrito produzido por 20 alunos voluntários, do Livro 3A (Nível B2 do CEFR), sendo 10 alunos no grupo de pesquisa e 10 alunos no grupo de controle (estudo experimental clássico). O total de alunos neste nível em 2011 é de 49, logo 41\% deles participam da pesquisa. Trata-se, portanto, de uma pesquisa de campo, empírica, uma vez que por sua natureza se caracteriza como um trabalho científico original, com objetivos descritivos (resultados diagnósticos), com alcance local, através de uma abordagem qualitativa, cuja coleta de dados perdurará por um período de 16 a 20 semanas, durante as quais os alunos produzirão semanalmente um texto, visando a atingir o total de 180 palavras por texto no final da pesquisa.

Será utilizado como instrumento de medida da evolução da habilidade de escrita dos alunos pertencentes ao estudo o CEFR, publicado pelo Conselho da Europa (Council of Europe). Este sistema é reconhecido internacionalmente e descreve a habilidade linguística em uma escala de seis níveis que vão de A1 para iniciantes a $\mathrm{C} 2$ para aqueles que possuem proficiência na língua estrangeira estudada. Será utilizado como base desta pesquisa o nível B2, que corresponde ao exame FCE dos testes aplicados pela Universidade de Cambridge. Serão praticadas as mesmas categorias de texto requeridas pela parte escrita do FCE, que são: artigo, e-mail, ensaio, carta, relatório, crítica, conto ou história ${ }^{20}$. Espera-se, para conseguir o diploma acima citado e como resultado desta pesquisa, que os alunos deste nível possam

\footnotetext{
${ }^{20}$ Os termos em inglês são respectivamente: article, email, essay, letter, report, review, story. Suas características diferem do que se conhece sobre suas traduções em português; por exemplo, o artigo (article) não tem relação com o artigo científico, mas com o artigo jornalístico, que neste contexto é um texto simplificado, comentando sobre assunto pré-determinado. Os textos produzidos devem conter entre 120-180 palavras de acordo com o exigido para o nível correspondente (B2) definido pelos parâmetros estabelecidos por Cambridge ESOL Exams e pelo CEFR.
} 
produzir textos claros e detalhados, expressando opiniões, desenvolvendo argumentações baseadas em diferentes pontos de vista (CEFR, 2009, p.27).

Os alunos serão diferenciados pelas letras maiúsculas A, se referindo a aluno, e A, B, C, etc., indicando uma sequência entre eles, para preservar seus nomes. A avaliação se pauta no desempenho do aluno, classificando aspectos tais como: conteúdo; organização e coesão; extensão de vocabulário e estruturas; precisão; registro; leitor alvo. São feitos breves comentários sobre cada item, e atribuída uma nota final, que varia de 0 para o desempenho menos satisfatório a 5 para o mais satisfatório, de acordo com aquilo que era pleiteado para a atividade. A aparente mudança quantitativa é, na verdade, uma mudança de ordem qualitativa dos mecanismos de ativação do conhecimento, que não atingirá seu ápice absoluto. (Logan, 1988; Vanlehn, 1996; Dekeyser, 2008).

\section{ANÁLISE DOS DADOS}

Ambas as teorias de aquisição de linguagem abordadas neste artigo tiveram sua origem na psicologia cognitiva, no behaviorismo e no conexionismo. Assim, existem entre elas muitas semelhanças na maneira de entender e promover o aprendizado. Serão tratadas a seguir algumas características presentes nestas teorias e sua aplicação ao estudo em desenvolvimento.

Como na primeira atividade realizada pelos alunos foram observadas interferências negativas da língua materna, buscou-se oferecer-lhes tanto input quanto intake, com o intuito de incentivar o aprendizado associativo, diminuindo, assim, a interferência negativa da L1 para que as experiências com a língua materna facilitem o aprendizado da L2 pela indução (Princípio emergente - CREED). Acrescentou-se o contexto, que contribui para a significação individual, combinando probabilidade, frequência e análise racional (Princípios Construtivo e Racional). Exemplos de interferência negativa: I won't go to visit [...] (AA e AG); It's great to read some from you (AF). Os alunos AA e AG utilizaram de maneira incorreta o futuro devido ao fato de construí-lo como o fariam na L1. O aluno AF empregou palavra de classe gramatical indevida no contexto, influenciado pela língua materna.

De acordo com o princípio dialético da mesma teoria, no processo de aquisição de uma língua o aprendiz se vê envolvido em uma tensão entre a sua interlíngua e o feedback recebido, o que pode levá-lo ao desenvolvimento social scaffolded, na tentativa de atingir uma comunicação mais elaborada e fluente, próxima daquela que o falante nativo possui. Podemos observar este aspecto nas construções do AL em diferentes atividades. Na primeira, ele teve 
dificuldade para adequar seu texto ao registro requerido, linguagem informal, usando muitas expressões formais da língua (conectivos como besides, lastly e construções indiretas) e cometendo erros como os acima citados, como por exemplo, $[. .$.$] you are finally making true$ your dream. A atividade (texto) recebeu classificação 2 de acordo com os padrões definidos pelo CEFR e utilizados pelo exame FCE. Nas atividades seguintes, ele obteve uma melhora qualitativa significativa. O segundo texto do aluno foi coerente e apropriado ao registro, novamente linguagem informal (carta), apresentando erros que não interferiam na comunicação, como uso inadequado de artigo, numerais e de poucas expressões com interferência negativa da L1. O aluno recebeu avaliação 4 pelo seu texto, resultado mantido nas atividades seguintes, mesmo em tipos de texto diferentes: artigo jornalístico, ensaio, entre outros.

A retroinformação oferecida (feedback) aos alunos variou de implícita a explícita. O grupo de pesquisa recebe comentários como os citados na sentença anterior, com variedade de estímulo a fim de incentivar a análise do material produzido. O grupo de controle recebe apenas a nota referente à avaliação de cada texto, e, raramente, um comentário genérico sobre suas dificuldades. A maioria dos alunos evoluiu qualitativamente na sua produção escrita e eles se mostraram satisfeitos com o feedback recebido. Em reunião feita com os dois grupos para avaliar o processo após a quarta semana de atividades, as insatisfações observadas foram poucas e referiram-se principalmente à falta de adaptação à ferramenta utilizada para veiculação e obtenção do material de apoio e retroinformação. Um aluno do grupo de controle demonstrou sua insatisfação com o feedback recebido, solicitando mais informações sobre as deficiências de seus textos. Houve remanejamento deste para o outro grupo. Outros dois alunos não estavam satisfeitos com a ferramenta utilizada; um deles, do grupo de controle, preferia trabalhar via computador e internet, e o outro, do grupo de pesquisa, não gostava de utilizá-los. O procedimento adotado foi o mesmo, e o resultado foi aumento da motivação e melhora no desempenho de todos eles.

Na mesma reunião, quando perguntados sobre características positivas e negativas do estudo, os alunos ressaltaram a motivação por aprender diferentes tipos de texto e a importância dos exemplos no aprendizado, traço condizente com a teoria de aquisição de habilidade e o princípio guiado pelo exemplo (CREED), segundo os quais a solução de problemas faz parte da aprendizagem e o exemplo facilita a inferência, criando correspondência entre problema e exemplo. Notou-se que alguns poucos alunos não evoluíram através da prática semanal, o que atribuímos à falta de análise da produção, dos próprios erros e/ou dos exemplos e princípios, não chegando a entendê-los de fato (Chi et al, 
1989). O AH, por exemplo, desenvolve o conteúdo satisfatoriamente, entretanto, tem dificuldade no uso da língua, produzindo períodos longos e confusos. A pontuação é deficiente, substituindo pontos por vírgulas: Sure I will be free on summer, I'll visit you, I'm dying to see Lady's puppies, they are so cute. Além disso, observa-se, algumas vezes, que ele não se atém ao exemplo e sugestões dadas para auxiliar a produção textual. Ele não consegue ultrapassar a classificação 3. Na primeira atividade, foi o único a não seguir a instrução de redigir uma carta informal negando um convite recebido, conforme exemplo fornecido.

Muitos alunos afirmaram ter mais dificuldade na escrita de textos formais e em lidar com assuntos que não lhes são familiares. Atribui-se esse fato à interferência positiva da língua materna (aprendizado associativo) e à frequência de regularidades, uma vez que eles são adolescentes e têm mais contato com a linguagem informal, possuindo conhecimento limitado sobre alguns temas. Afirmaram gostar de fazer consultas a dicionários e hipertextos para obter informações mais precisas acerca de assuntos sobre os quais não possuem conhecimento aprofundado e fazer pesquisas sobre estruturas e vocabulários que lhes geram dúvidas. O AQ compara escrita e fala, considerando a primeira mais difícil que a segunda, e a escrita em L1 mais fácil que em L2.

Como se busca atingir o estágio automatizado, preconizado pela teoria de aquisição de habilidade, deseja-se observar os efeitos da prática, isto é, aumento de velocidade e precisão. Até este momento da pesquisa, ainda se nota a presença de erros que se acreditam consequência da falta de conhecimento, situação na qual o aprendiz opta por não procurar ajuda, seja ela de qualquer natureza, e inventar o que lhe convier no momento da execução da tarefa a fim de dar-lhe continuidade (Vanlehn, 1990, 1995 apud Vanlehn, 1996). A seguir listamos alguns exemplos de erros que se supõem decorrentes da falta de conhecimento. AK: [...] the beaches and others summer's things [...]; [...] and I must to say that [...] (provavelmente ignora o uso correto do possessivo adjetivo e do adjetivo no primeiro fragmento, e do modal no segundo); AM: [...] you and Alex can stay a few days here at my home, don't you? (uso incorreto de tag questions). Palavras inventadas: attenciously (AI); [...] I am a very good cooker (AO); [...] the ambient is just perfect and the food ... simply awesome (AM).

Buscamos analisar, neste artigo, os casos considerados mais significativos que emergiram até o presente momento da pesquisa, e que são exemplares para ilustrar o propósito da pesquisa. Naturalmente, há outras ocorrências que não foram aqui abordadas e serão devidamente analisadas com o desenrolar da pesquisa. 


\section{CONSIDERAÇÕES FINAIS}

Partindo do pressuposto de que a habilidade de escrita é muitas vezes negligenciada no ensino de Inglês como língua estrangeira, buscou-se minimizar essa defasagem através da ênfase na prática além das limitações impostas pelo dia-a-dia da sala de aula. O CALL aparece como aliado para proporcionar estímulos, promover a interação e o desenvolvimento da competência linguística. A variedade dos tipos de texto e a prática intensiva visam à motivação, à melhoria da argumentação, à redução de erros e à consequente automatização da habilidade.

Até o presente momento o resultado tem sido satisfatório para ambas as partes envolvidas na pesquisa: os alunos apresentaram motivação e desenvolvimento rápido e considerável, os pesquisadores conseguiram detectar problemas e trabalhar soluções para resolvê-los, ainda que parcialmente. As teorias utilizadas como suporte para o estudo se mostraram eficazes no seu propósito, o que pode ser comprovado pelos exemplos e pela evolução da qualidade do material produzido, embora se pretenda continuar a pesquisa com o intuito de conseguir resultados mais abrangentes e dados mais consistentes para análise.

\section{REFERÊNCIAS}

Anderson, J. R. (1982). Acquisition of Cognitive Skill. Psychological Review, 89 (4), 369406.

Anderson, J. R. (1993). Rules of the mind. Hillsdale, NJ: Lawrence Erlbaum Associates.

Anderson, J. R. et al (2004). An integrated theory of the mind. Psychological Review, 111, 1036-1060.

Brown, H.D. (2007). Teaching by Principles: An Interactive Approach to Language Pedagogy. United States of America: Longman/Pearson.

Byrne, D. (Ed.). (1986). Teaching oral English. Harlow, UK :Longan.

CAMBRIDGE UNIVERSITY PRESS. Common European Framework of Reference for Languages: Learning, Teaching, Assessment. Disponível em <http://www.coe.int/lang> Acesso em: 26 de out. 2010.

CAMBRIDGE UNIVERSITY PRESS - ESOL EXAMINATIONS. First Certificate in English: Handbook for Teachers for Examinations from December 2008. Reino Unido, 2008. Disponível em <http://www.cambridgeesol.org/fce> Acesso em: 01 de nov. 2010 
Celce-Murcia, M. (2001). Teaching English as a Second or Foreign Language. United States of America: Heinle \& Heinle Thomson Learning.

Chapelle, C.A. (2009). The Relationship Between Second Language Acquisition Theory And Computer-Assisted Language Learning. Modern Language Journal, 93 (1), 741-753, 2009.

Chi M. T. H. et al. (1989). Self-explanations: how students study and use examples in learning to solve problems. Cognitive Science. 15 (1), 45-182.

COUNCIL OF EUROPE. Relating Language Examinations to the Common European Framework of Reference for Languages: Learning, Teaching, Assessment (CEFR). Cambridge: Cambridge University Press. (Janeiro 2009). Disponível em <http://www.coe.int/lang > Acesso em: 26 de out. 2010.

Crystal, D. (2010). The Cambridge Encyclopedia of Language. United Kingdom: Cambridge University Press.

Davies, P. A.; Falla, T. (2008). FCE Result: Student's Book. Oxford University Press.

Dekeyser, R. (2008). Skill Acquisition Theory. In Vanpatten, B. \& J. Williams (Eds.) Theories in Second Language Acquisition: An Introduction. NJ: Lawrence Erlbaum, 97-114.

Doughty, C. J.\& Long, M. H. (Ed.). (2004). The Handbook of Applied Linguistics. United Kingdom: Blackwell Publishing.

Ellis, N. C. (2006). Cognitive Perspectives on SLA: The Associative-cognitive CREED. AILA Review, 19 (1), 100-121.

Ellis, N. C. (2008). The Associative Cognitive CREED. In: Vanpatten, B. Input Processing in Adult Second Language Acquisition. In Vanpatten, B. \& J. Williams (Eds.) Theories in Second Language Acquisition: An Introduction. NJ: Lawrence Erlbaum, 77-95.

Ellis, R. (2003). Second Language Acquisition. Honk Kong: Oxford University Press.

Fitz, P., \& Posner, M. (1967). Human Performance. Belmont, CA :Brooks/Cole.

Freitas, M. T. A; Costa, S. R. (org.). (2006). Leitura e Escrita de Adolescentes na Internet e na Escola. Belo Horizonte: Autêntica.

Garrett, N. (2009a) Technology in the Service of Language Learning: Trends and Issues. The Modern Language Journal, 93, 697-718.

Garrett, N. (2009b). Computer-Assisted Language Learning Trends and Issues Revisited: Integrating Innovation. The Modern Language Journal, 93, 719-740.

Lafford, B.A. (2009). Toward an Ecological CALL: Update to Garrett. The Modern Language Journal, 93, 673-696. 
Logan G. D. (1988). Toward an Instance Theory of Automatization. Psychological Review, 95(4), 492-527.

Selinker, L. (1972). IRAL - International Review of Applied Linguistics in Language Teaching, 10 (1), 209-232

Norris, R. (2008). Ready for FCE: Coursebook with key. Macmillan Exams.

Otto, S. E. K. \& Pusack, J. P. (2009). Computer-Assisted Language Learning Authoring Issues. The Modern Language Journal, 93 (1), 784-801.

Richards, J.C. \& Schmidt, R. (2002). Longman Dictionary of Language Teaching \& Applied Linguistics. Malaysia: Pearson Education Limited.

Skinner, B. F. (1989). The Origins of Cognitive Thought. Recent Issues in the Analysis of Behavior.

Taylor, L. \& Jones, N. Cambridge ESOL exams and the Common European Framework of Reference (CEFR), Research Notes, 24 (1), maio de 2006. Disponível em <http://www.cambridgeesol.org/exams> Acesso em: 23 de out. 2010.

The Alte Can Do Project: English Version. 2002. Disponível em <http://www.coe.int/lang> Acesso em: 26 de out. 2010.

Vanlehn K. (1996).Cognitive Skill Acquisition. Annal Review of Psychology, 47 (1), 513539.

Vanpatten, B. (2008). Input Processing in Adult Second Language Acquisition. In Vanpatten,B. \& Williams J. (Eds.) Theories in Second Language Acquisition: An Introduction. NJ: Lawrence Erlbaum, 115-136.

\section{OS AUTORES}

Sheila Cristina Alves de Ávila é graduada em Letras pela UFSJ (1996) e mestranda em Ciências da Educação pela Universid Americana - Assunção (Iniciado em 2010). Tem experiência na área de Letras, com ênfase em Linguística Aplicada e ensino de Língua Estrangeira, atuando principalmente nos seguintes temas: teorias de aprendizagem e CALL. Professora do Ensino Básico Federal (EPCAR-Barbacena-MG)

E-mail: sheila-avila@hotmail.com

Anderson Luiz da Silva possui graduação em Letras pela UFJF (1994), mestrado em Letras (1998) e doutorado em Letras (2006) pela PUC-Rio. Tem experiência nas áreas de Letras e Pedagogia, com ênfase em Literatura Brasileira, atuando principalmente nos seguintes temas: intertextualidade, prosa de ficção do século XX, literatura/antropologia, formação de professores.

Professor do Ensino Básico Federal (EPCAR-Barbacena-MG)

E-mail: andersonsilvamg@yahoo.com.br 